\title{
Gergis und Marpessos in der Troas.
}

\section{Von Richard Kiepert.}

Ueber die Stadt Ger $\mathrm{g}$ is in der Troas liegen folgende Angaben vor. Zuerst nennt Herodot V, 122 und VII, 23 die Einwohner Gergithes und bezeichnet sie als Reste der alten Teukrer oder Troer. Die zweite Stelle ist für die Festlegung des Ortes wichtig: Xerxes erreicht, von Süden her, von Adramyttion und Antandros kommend, den Skamandros, zieht nach dem Pergamon des Priamos (heute Hisarlik) hinauf, opfert dort und marschiert am folgenden Tage weiter nach Abydos, wobei er links Rhoiteion (Palaeokastra), Ophryneion (Ruinen bei Eren Köi) und Dardanos (Ruinen $2 \mathrm{~km}$ südlich rom Vorgebirge Kefez Burnu), rechts aber die Stadt der Gergithes zu liegen hatte. Daraus folgt, dass Gergis von der nach Nordosten verlaufenden Linie Rhoiteion-Ophryneion-Dardanos landeinwärts $d$. h. etwa in südöstlicher Richtung zu suchen ist, unbestreitbar $\mathrm{n} \ddot{\mathrm{r}} \mathrm{d}$ li ch des Skamandros-Flusses. Nun hat Calvert (Archaeological Journal XXI, 1864, 48 ff.) Gergis in den Ruinen des Ballyk Dagh, $10 \mathrm{~km}$ südsüdöstlich von Hisarlik auf dem linken Skamandrosufer wiederzuerkennen geglaubt und die Zustimmung von Eduard Meyer (Geschichte von Troas S. 23) und Judeich (Kiepert-Festschrift S. 226 und Sitzungsber. Akad. d. Wissensch. Berlin 1898, S. 540 f.) gefunden. Aber Xerxes hatte, als er in nordöstlicher Richtung bei jenen drei Städten vorbeizog, den Ballyk Dagh, wie ein Blick auf die Karte zeigt, genau im Rü cken und nicht zur rechten Seite. Der $17 \mathrm{~km}$ von Alexandreia Troas entfernte Ballyk Dagh kommt mithin für Gergis, das nach Pausan. X, 12, 4 (s. unten) mindestens 200 bis 220 Stadien d. h. $36-39 \mathrm{~km}$ von Alexandreia Troas entfernt war, nicht in Betracht; H. Kiepert hat auf seiner Karte Asia Provincia (Formate Orbis Antiqui IX und Text S. 3, Anm. 31) dort $\mathrm{P}$ et $\mathrm{r}$ a eingetragen, das in einem Briefe des Antiochos I Soter an Meleagros vorkommt (Dittenberger, Sylloge $\left.{ }^{1}, 158\right)$; der Name passt gut zur Lage.

Nach Xenophon Hellen. III, 1, $10 \mathrm{ff}$. verwaltete die Dardanerin Mania unter Pharnabazos ein kleines Vasallenreich, zu dem die Städte Skepsis (auf dem Kursehunlu Tepe nördlich vom oberen Skamandros), Kebren (auf dem Fughla Tepe $14 \mathrm{~km}$ WSW von den Ruinen von Skepsis und siidlich 
vom Skamandros), Gergis und später die Küstenstädte Larisa (südlich von Alexandreia Troas), Kolonai (bei Köse Deresi) und Hamaxitos (am Gök Tepe) gehörten. Diese Ortsliste zeigt, dass Gergis unweit von Skepsis und Kebren gelegen hat. Der schon erwähnte Brief des Antiochos weist sodann den Meleagros an, 2000 Plethren Ackers von dem an das Gebiet von Gergis oder Skepsis angrenzenden Lande einem gewissen Aristodikides anzuweisen und es dem Gebiete von Ilion oder Skepsis anzugliedern. Ilion liegt nun $10 \mathrm{~km}$, Skepsis 38 vom Ballyk Dagh entfernt; hätte dort auf dem Ballyk Dagh Gergis gestanden, so wäre Meleagros wohl ange-

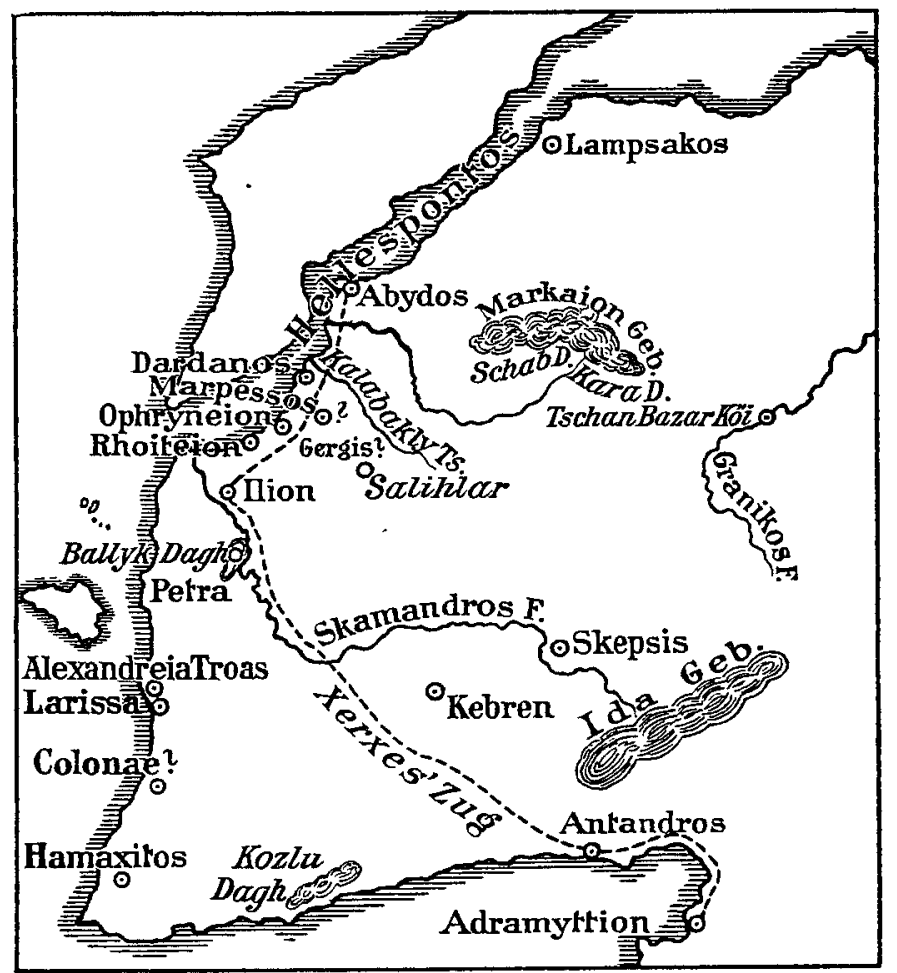

wiesen worden, die 2000 Plethren dem nahen ilischen Gebiete zuzuschlagen und ihm nicht die Wahl zwischen llion und dem fast viermal so weit entfernten Skepsis gelassen worden. Gergis muss also weiter landeinwärts, näher an Skepsis gesucht werden, als auf dem Ballyk Dagh.

Stephanus Byzantinus s. $\Gamma^{\prime}$ '́oyıs bietet nichts geographisches; nur gibt er an, dass der Genetiv $\Gamma \dot{\varepsilon} 0 \gamma \iota \vartheta$ gebraucht werde, und dass Gergithia die weissagende Sibylle heisse, deren

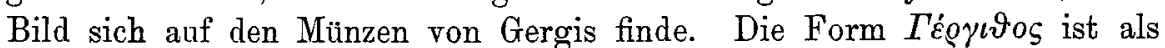
Gergithus bei Livius 38, 3910 zu finden: Iliensibus Rhoeteum et Gergithum addiderunt (Romani), woraus sich wiederum die $\mathrm{Nach} \mathrm{bar-}$ 
schaft von Ilion und Gergis ergibt. Die Schol. Plat. Phaedr.

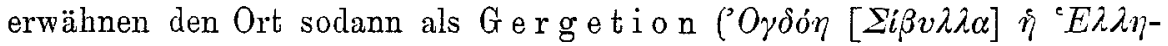

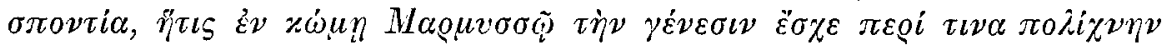

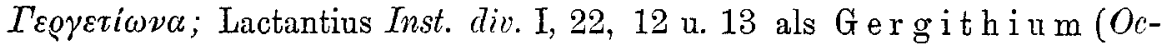
tavam Hellespontiam in agro Troiano natam vico Marmeso circa oppidum Gergithizm) und Suidas s. Si $\beta v \lambda \lambda \alpha$ (Bernhardy II, 2, 742) als G e rgit-

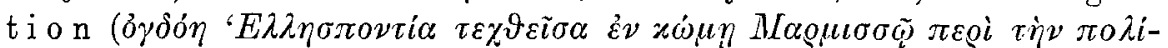

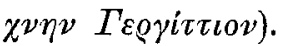

Weiter in der topographischen Bestimmung von Gergis führt uns die Sichtung der Namen, mit welchen die alten Autoren das in der $\mathrm{N} \ddot{a}$ h e von $G$ e r $g$ i s gelegene Heimatsdorf der Hellespontischen Sibylle bezeichnen. M armyssos heisst es in den Scholien zu Platon (s. oben), Marmissos bei Suidas s. Si $\beta v \lambda \lambda a$ (s. oben), Marmessus bei Lactantius (s.

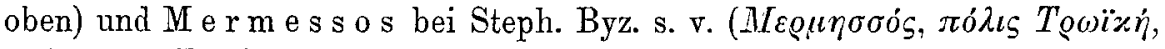

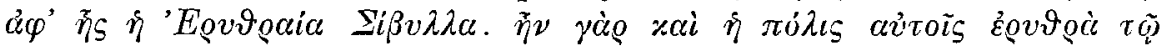
$\chi \varrho \omega ́(\tau \tau \iota)$. Zweimal dagegen wird es $\mathrm{M}$ a r p e s s o s genannt, von Tibull. II, 5. 67 (Quidquid Amalthea, quidquid Marpesia dixit) und von Pausanias X, 12, 4, topographisch der wichtigsten Stelle. Nachdem er ein Orakel der Sibylle mitgeteilt, welches mit den Worten schliesst

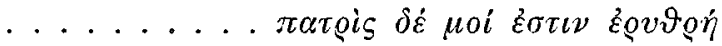

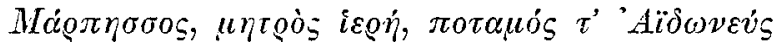

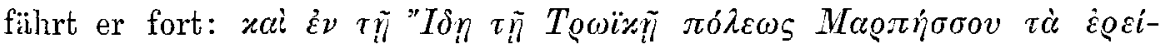
$\pi \iota \alpha \ldots$. .

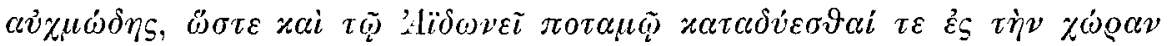

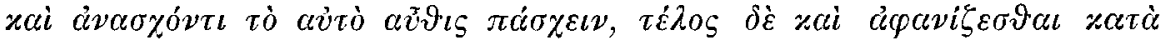

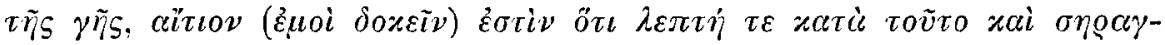

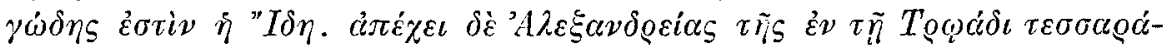

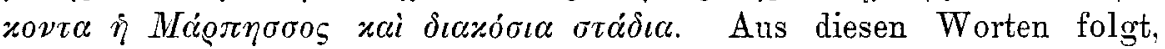
dass des Pausanias Marpessos identisch mit dem Mermessos des Steph. Byz. ist, weil beide, wie auch das Orakel, das Dorf als Heimat der Sibylle bezeichnen und die rötliche Erde als besonderes Kennzeichen hervorheben. Vorzüglich wertvoll ist aber die Angabe, dass Marpessos 240 Stadien $(43,2 \mathrm{~km})$ von Alexandreia Troas entfernt war: diese bringen uns, wenn wir, wie üblich, etwa ein Sechstel für die Biegungen und Steigungen des Weges abziehen, also $36 \mathrm{~km}$ rechnen und die aus Herodot VII, 23, Xenophon, dem Briefe des Antiochos und Livius gewonnenen ungefïhren Andeutungen über die Lage des nahen Gergis damit kombinieren, in die Gegend nordwestlich von Salihlar (21 km östlich von Tlion) oder auch näher zur Küste, wo H. Kiepert's Karte vom Westl. Kleinasien Bl. IV $3 \mathrm{~km}$ nordöstlich von der Stätte von Ophryneion Ruinen angibt, die in der Luftlinie genan $36 \mathrm{~km}$ von Alexandreia Troas entfernt sind. Dass Pausanias dieses Hügelland zum Ida-(xebirge rechnet, kann nicht dagegen sprechen; bei den höchst unvollkommenen Karten der Alten konnte er 
es wohl für einen Ausläufer des Ida halten. In Wirklichkeit hat aber die "fürchterlich“ dürre. poröse Umgegend von Marpessos und ihr versickernder und schliesslich ganz unter der Erde verschwindender AïdoneusFluss mit dem waldreichen Ida, dessen ständiges Beiwort in der Ilias $\pi 0$ $\lambda v \pi i \delta \alpha \xi$ ist, nichts zu tun.

Aber für die Wiedera uffind ung von Gergis, Marpessos und Aïdoneus kann die Beschreibung des Pausanias von dem rötlichen trockenen Erdboden und dem unter der Erde verschwindenden Flusse von Bedeutung werden, wenn sich einmal einer der zahlreichen Troja besuchenden Altertumsfreunde entschliessen sollte, von der Völkerstrasse des Hellespont aus einige Meilen weit in das Innere vorzudringen, um mit eigenen Augen zu sehen. Konnte doch auf solche Art Judeich (Jahreshefte Oest. Arch. Inst. IV, 124) H. Kiepert's Vermutung, Marpessos entspreche den Ruinen auf dem Kozlu Dagh an der troischen Südküste (Formae Orbis Antiqui IX, Text, S. 3), durch die einfache Beobachtung abweisen, dass das dortige Gestein nicht rot gefärbt, sondern graubrauner Trachyt ist.

Verschieden von dem in der Gegend von Salihlar zu suchenden Gergis ist das weinberïhmte Ge r $\mathrm{g}$ ith $\mathbf{i}$ on in der Lampsakene (Strab. 589

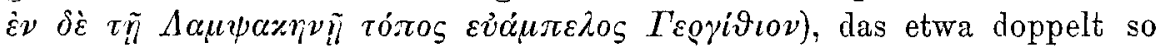
weit von Alexandreia Troas entfernt war, als das erste. Vielleicht ist es identisch mit dem Gergis, das Steph. Byz. s. Máoxoıov erwähnt:

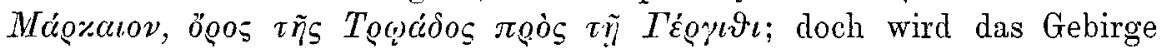
nur bier genannt, so dass es nur eine allerdings wahrscheinliche Vermutung ist, dass es den heutigen Kara Dagh und Schab Dagh zwischen den Granikosquellen und dem Hellesponte entspreche. Ed. Meyer (Geschichte ron Troas 23), Lolling (bei J. Müller, Handluecth der kilass. Altertumswiss. III, 250; er wirft die beiden Gergis zusammen) und H. Kiepert (Formae 0. A. IX) suchen dies Gergis am oberen Granikos, etwa bei Tschan Bazar Köi. Dies muss aber dahingestellt bleiben; denn wir wissen nicht, ob das Gebiet von Lampsakos so weit landeinwärts gereicht hat. Vielleicht ist das antike Weinland der Lampsakene eher auf dem hellespontischen Abhange des Marlaion zu suchen: durch heutige Verhältnisse lässt sich das nicht mehr entscheiden, denn der treffliche Wein von Lampsakos, dessen sich Themistokles durch des Perserkönigs Gnade erfreute, soll heute einem abscheulichen Getränke Platz gemacht haben.

Schliesslich gab es auch bei diesem Gergis einen Ort, der ähnlich dem oben besprochenen Marmyssos, Marmissos, Marmessos, Mermessos

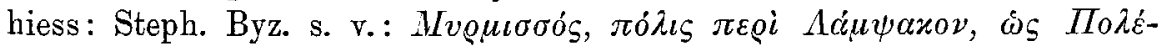
$\mu \omega \nu$. Seine Lage ist ebenso unbekannt, wie die des nördlichen Gergithion. Berlin. 